\title{
Chapitre VI - Typologie et situation financière des entreprises de TV et de TVB
}

Maurice Wolkowitsch

\section{CpenEdition}

\section{Journals}

Édition électronique

URL : https://journals.openedition.org/rhcf/1408

DOI : 10.4000/rhcf.1408

\section{Éditeur}

Rails \& histoire

\section{Édition imprimée}

Date de publication : 10 février 2004

Pagination : 196-221

ISBN : 0996-9403

ISSN : 0996-9403

\section{Référence électronique}

Maurice Wolkowitsch, "Chapitre VI - Typologie et situation financière des entreprises de TV et de TVB », Revue d'histoire des chemins de fer [En ligne], 30 | 2004, mis en ligne le 24 août 2012, consulté le 22 avril 2022. URL : http://journals.openedition.org/rhcf/1408 ; DOI : https://doi.org/10.4000/rhcf. 1408 


\section{Chapitre VI \\ Typologie et situation financière des entreprises de TV et de TVB}

es TV et TVB ont pour originalité, au sein des réseaux
secondaires, de s'inscrire prioritairement dans des milieux urbanisés, d'avoir les communes et les syndicats de communes pour autorité de tutelle, d'utiliser un matériel moins diversifié que les CFIL et TVM, d'être enfin dans une situation financière particulière. Les TV et TVB sont soumis pour leur établissement à des procédures proches des autres VFIL ; ils subissent la concurrence automobile qui engendre les mêmes difficultés et les mêmes évolutions, pouvant conduire à la mort des réseaux ferrés et, parfois, à celle des entreprises.

De puissantes sociétés se sont constituées, malgré la dispersion territoriale des réseaux, soit pour prendre le contrôle de réseaux sur le plan financier, soit pour en assurer l'exploitation directe. Elles ne sont pas seules a exercer cette activité : d'autres entreprises sont demeurées dans un cadre municipal. Une évolution s'est produite au cours du temps.

Une centaine d'entreprises gère les réseaux de TV et de TVB, les premiers représentant les deux tiers de l'ensemble. La coexistence au sein d'une même entreprise de lignes urbaines de TV et suburbaines de TVB est banale. Des compagnies de CFIL et de TVM exploitent quelquefois des lignes de TV ou de TVB. Si le kilométrage total exploité par des compagnies ayant des lignes sous deux statuts est réparti à égalité entre eux, il en va autrement au sein de chacune d'elles, puisque la part des TV y varie de 3,8 à $92 \%$ (tabl. 32). Attachons-nous à mieux connaitre les entreprises.

\section{Les entreprises à l'origine des réseaux de TV}

\section{. Les premières entreprises}

À l'origine, des entreprises se sont créées pour obtenir la concession d'un réseau à exploiter dans des limites communales avec des extensions éventuelles dans les circonscriptions voisines. Après un temps d'exploitation, certaines de ces entreprises ont connu une situation financière difficile, elles n'ont pu satisfaire la demande. Cette incapacité conduisait à un changement de statut. Le capital de départ était parfois modeste, comme à Rouen ou à Poitiers (annexe 1) mais il pouvait égaler 
Tableau 32. Place du réseau de TV ou TVB dans les entreprises exploitant des lignes sous un autre statut en \% du kilométrage exploité

\begin{tabular}{|c|c|c|c|}
\hline Compagnies & Km TV & Km TVB & $\%$ TV $/$ total \\
\hline Compagnie de tramw ays électriques d'Angers & 14 & 16 & 47 \\
\hline Compagnie de tramw ays électriques de Dijon & 16 & 10 & 61 \\
\hline $\begin{array}{l}\text { Compagnie de tramw ays électriques de } \\
\text { Rouen }\end{array}$ & 69 & 6 & 92 \\
\hline $\begin{array}{l}\text { Société versaillaise de tramw ays électriques } \\
\text { et de distribution d'électricité }\end{array}$ & 16 & 5 & 76 \\
\hline Compagnies & Km TV & Km TVM & $\% \mathrm{TV} /$ total \\
\hline $\begin{array}{l}\text { Compagnie de chemin de fer de Pau à Oloron } \\
\text { et Mauléon et du tramw ay de Bayonne à } \\
\text { Biarritz }\end{array}$ & 9 & 230 & 3,8 \\
\hline Compagnie des tramw ays de Tours & 14 & 22 & 39 \\
\hline $\begin{array}{l}\text { Compagnie des tramw ays électriques de } \\
\text { Béziers }\end{array}$ & 10 & 13 & $43(a)$ \\
\hline Compagnie des tramw ays de Douai & 16 & 5 & $76(a)$ \\
\hline $\begin{array}{l}\text { Compagnie des tramw ays électriques de Lille } \\
\text { et sa banlieue }\end{array}$ & 11 & 92 & 10 \\
\hline Compagnies & Km TV & $\begin{array}{c}\mathrm{Km} \\
\text { CFIL1880 }\end{array}$ & $\% \mathrm{TV} /$ total \\
\hline Compagnie des omnibus et tramw ays de Lyon & 238 & 33 & 88 \\
\hline Compagnies & $\mathrm{Km}$ TVB & Km TVM & $\% \mathrm{TV} /$ total \\
\hline Compagnie des tramw ays de Nice et du littoral & 81 & 60 & 57 (b) \\
\hline Société grenobloise de tramw ays électriques & 46 & 15 & 75 \\
\hline Société des tramw ays bretons & 7 & 17 & 29 (c) \\
\hline \multicolumn{4}{|c|}{$\begin{array}{l}\text { (a) Mise en service en } 1898 \text { à Douai et } 1901 \text { à Béziers ; en } 1911 \text { ces } \\
\text { compagnies n'avaient .pas réussi à créer un trafic de marchandises prévu dans } \\
\text { l'acte de concession. } \\
\text { (b) Compagnie figurant dans le tabl. } 15 \text { au titre de TVM. } \\
\text { (c) Ligne de Saint-Malo à Cancale exploitée en TVB de Saint-Malo à Paramé, en } \\
\text { TVM au-delà. }\end{array}$} \\
\hline
\end{tabular}


celui d'une compagnie de CFIL ou de TVM : la Compagnie des tramways de Nîmes n'a rien à envier à la Compagnie des chemins de fer du Périgord (tabl. 33). Deux entreprises ont regroupé chacune deux réseaux : l'Ouest électrique avec Rennes (16 km) et Le Mans (11 km), la Compagnie centrale de tramways électriques, avec Perpignan $(25 \mathrm{~km})$ et Angoulême (16 km). L'exploitation d'un ou de deux réseaux ne différenciait guère ce type d'entreprises : leur siège social était à Paris, rarement, comme à Rouen, dans la ville où elles exploitaient un réseau ; leurs administrateurs étaient parisiens ou lyonnais, la participation de notables locaux était encore plus faible que dans le cas des CFIL; ces administrateurs parisiens se retrouvaient dans plusieurs conseils d'administration, tels Burrel et Thurnauer en 1911 pour les compagnies de tramways de Rouen et de Nice et du littoral ; simples intérêts personnels ou traduction de liens plus subtils entre les entreprises ?

Tableau 33. Les entreprises de TV et TVB : le capital engagé en 1911

\begin{tabular}{|c|c|c|c|c|c|c|c|c|}
\hline Statut & $\begin{array}{c}\text { Com pa- } \\
\text { gnies }\end{array}$ & Km & $\begin{array}{c}\text { Fonda- } \\
\text { tion }\end{array}$ & $\begin{array}{c}\text { Nom bre } \\
\text { d'actions }\end{array}$ & $\begin{array}{c}\text { Prix de } \\
\text { l'action à } \\
\text { l'ém ission }\end{array}$ & Capital & Siège & $\begin{array}{c}\text { Rés idence } \\
\text { des adm inis- } \\
\text { trateurs }\end{array}$ \\
\hline TV & $\begin{array}{c}\text { Tramw ays } \\
\text { de Rouen }\end{array}$ & 75 & 1875 & 27000 & 500 & $13,5 \mathrm{MF}$ & Rouen & 6 Paris \\
\hline TV & $\begin{array}{c}\text { Tramw ays } \\
\text { de Royan }\end{array}$ & 15 & 1896 & 39000 & 125 & $4,825 \mathrm{MF}$ & Paris & $\begin{array}{c}3 \text { Paris, } \\
2 \text { Royan }\end{array}$ \\
\hline TVB & $\begin{array}{l}\text { Tramw ays } \\
\text { de Poitiers }\end{array}$ & 4 & 1896 & 10000 & 100 & $1 \mathrm{MF}$ & Lyon & $\begin{array}{c}2 \text { Paris, } \\
2 \text { Lyon, } \\
1\end{array}$ \\
\hline TV & $\begin{array}{c}\text { Tramw ays } \\
\text { de Nîmes }\end{array}$ & 15 & 1898 & 6000 & 500 & $3 \mathrm{MF}$ & Lyon & 5 Lyon \\
\hline
\end{tabular}

\section{. Quatre grandes entreprises}

L'intérêt des milieux financiers pour l'exploitation de réseaux de tramways s'est concrétisé dans la constitution de puissantes compagnies, sans doute attirées par l'idée que la rentabilité pouvait être supérieure à celle des longues lignes rurales; ces compagnies sont fondées entre 1875 et 1911 ; leur développement est lié à celui du tramway électrique. Certaines compagnies partent sur des bases limitées, mais des augmentations de capital successives en feront des puissances financières, d'autres rassemblent dès leur constitution des capitaux supérieurs à ceux de la SGCFE ou des CFD. Ces entreprises recherchent des concessions 
à Paris ou dans les grandes villes de province ou se proposent de contrôler une multitude de réseaux ; leur stratégie est voisine de celle du groupe Empain, à une autre échelle : est-ce un hasard si plusieurs d'entre elles comptent des Belges dans leur conseil d'administration ? Les entreprises contrôlées conservent au moins les apparences de l'indépendance à travers leur conseil d'administration. Les grandes entreprises ont une politique de diversification de leurs activités avec une prédilection pour le domaine de l'électricité, production et distribution; n'oublions pas que les années vingt sont celles de l'électrification rurale appuyée sur de petites sociétés de distribution. Les données relatives aux quatre entreprises étudiées correspondent à 1927, sauf mention contraire dans le texte ${ }^{1}$.

La Compagnie générale française de tramways remonte à 1875 , elle ajoutera le mot «électrique » le moment venu. Un capital de $55 \mathrm{MF}$ divisé en 110000 actions de $500 \mathrm{~F}$ est réuni dès l'origine. Le siège social est parisien. Les administrateurs résident pour neuf d'entre eux à Paris, un à Versailles, un à Liège. En 1911, la compagnie exploitait $264 \mathrm{~km}$ : 164 à Marseille, 46 au Havre, 32 à Nancy, 23 à Orléans. En 1927, le réseau atteint $334 \mathrm{~km}$; en outre, les réseaux de tramways de SaintQuentin $(9 \mathrm{~km})$, Toulon $(75 \mathrm{~km})$ et Tunis $(105 \mathrm{~km})$ sont des « affaires domiciliées » (annexe 2).

L'Omnium lyonnais est une entreprise née à Lyon où elle a son siège, en 1896. Six de ses administrateurs sont parisiens, un est marseillais, un bellifontain. L'entreprise a une histoire financière complexe ; elle commence petitement : en janvier 1896 le capital est de $750000 \mathrm{~F}$ divisé en 7500 actions de $100 \mathrm{~F}$; des augmentations successives portent le capital à 1,5 MF en juillet 1896, $4 \mathrm{MF}$ en août 1897, $10 \mathrm{MF}$ en août 1898 et $20 \mathrm{MF}$ en mars 1900, avec une émission de 200000 actions de $100 \mathrm{~F}$, vendues au cours de $114 \mathrm{~F}^{2}$. Cette rapide montée en puissance illustre l'attrait qu'exerçait sur la bourse l'exploitation de réseaux de tramways; il est vrai que l'Omnium lyonnais s'intéressait à d'autres activités. La compagnie exploitait huit réseaux de tramways, en tout $87 \mathrm{~km}^{3}$, elle avait des intérêts dans quatre entreprises de transport ${ }^{4}$

1- Annuaire des chemins de fer et des tramways, ancien Marchal, édition des Réseaux français, Paris, 1927

2- Une nouvelle opération ramène le capital à $10 \mathrm{MF}$ avec échange de deux actions anciennes contre une nouvelle.

3- Avignon, Bourges, Cannes, Cette, Fontainebleau, Pau, Poitiers, Troyes.

4- Chemin de fer électrique souterrain nord-sud de Paris, Compagnie genevoise de tramways électriques, Chemin de fer à voie étroite de Firminy à Saint-Étienne et Rivede-Gier, Chemin de fer sur routes d'Algérie. 
et quatre entreprises industrielles dont deux tournées vers l'électricité5. L'Omnium lyonnais était bien devenu un groupe (annexe 3).

La Société parisienne pour l'industrie des tramways et chemins de fer électriques naît en 1900 avec un capital de 25 MF, égal à celui de la SGCFE, divisé en 100000 actions de $250 \mathrm{~F}$; dès 1901, le capital est porté à $65 \mathrm{MF}$. Le siège social est à Paris. Huit administrateurs résident à Paris, deux sont belges et résident à Bruxelles, le général baron Empain et François Empain. Les entreprises dans lesquelles la société est " notamment intéressée », suivant la formule consacrée, sont celles que nous avons déjà reconnues comme faisant partie du groupe Empain (CVF, Chemins de fer du Calvados, Chemins de fer économiques du Nord, Chemins de fer métropolitain de Paris) auxquelles il convient d'adjoindre les tramways de Lille, de Paris et du département de la Seine. Le mot «notamment » indique que la liste n'est pas exhaustive. Cette société paraît comme la branche française des organismes financiers du groupe ; elle a, entre autres, l'objectif de financer «les constructions des chemins de fer, tramways et omnibus... ».

La société dénommée «Les Exploitations électriques » est la plus jeune : 1911. Son capital originel de 10 MF divisé en 40000 actions de $250 \mathrm{~F}$ est porté successivement à 12,5 MF en 1914, 18 en 1922, 25 en 1924. Neuf des administrateurs sont bruxellois, trois parisiens, trois lyonnais, un domicilié à Boulogne-sur-Seine. Le siège social est à Paris. La société contrôle cinq réseaux de distribution d'électricité ${ }^{6} ; 21$ réseaux de tramways ${ }^{7}$ sont, à son siège, des affaires domiciliées ; la liste comprend Le Mans et Rennes, réseaux exploités en 1911 par l’Ouest électrique, intégré à la Société bretonne d'électricité, elle-même contrôlée par «Exploitations électriques ».

Les réseaux des régions lilloise, marseillaise et stéphanoise étaient liés à l'une ou à l'autre de ces compagnies ; ceux d'autres villes importantes (Bordeaux, Nice... ) conservaient leur réseau indépendant. Plus d'une trentaine d'entreprises de moindre envergure entretenait d'une façon ou d'une autre des relations avec les principales sociétés ; elles pouvaient leur affermer l'exploitation de leur réseau : la domiciliation

5- Société des forges motrices de la Sélune, Société électrique de la sidérurgie lorraine, Société minière française au Maroc, Société des mines de Boudjoudoun.

6- Société bretonne d'électricité, Union des producteurs d'électricité des PyrénéesOrientales, Énergie électrique du Rouergue, Electro mecanico industrial, Électricité d'Alep.

7- Angers, Angoulême, Besançon, Béziers, Brest, Caen, Châlons-sur-Marne, Cherbourg, Dijon, Lorient, Le Mans, Mézières, Montpellier, Nîmes, Oran, Perpignan, Rennes, Saint-Étienne, Sedan, Tours, Tours à Vouvray. 
était une autre forme de collaboration, pouvant aller de la simple disponibilité d'une adresse à la formation d'intérêts convergents. Seule la lecture de chaque contrat permettrait d'apprécier la nature des relations établies. Faute d'en disposer, l'évolution dans la composition des conseils d'administration peut en donner une indication.

La Compagnie des tramways de Poitiers a en 1911 le comte Benédetti parmi ses cinq administrateurs ; le même en 1927 est entré au conseil de l'Omnium lyonnais, qui exploite le réseau de cette ville; plus significatif, en 1936, le conseil de la Compagnie des tramways de Poitiers, désormais fort de dix membres, compte dans ses rangs quatre administrateurs de l'Omnium lyonnais, dont le président de cette société 8 . Cette manifestation de liaisons financières se lit encore avec le passage d'un administrateur de la Compagnie générale française de tramways en 1927 à la Compagnie des tramways de Rouen en 1936, M. Goury du Roslan.

Certains réseaux, qui paraissaient moins susceptibles de forte rentabilité, sont demeurés apparemment à l'écart de ces mouvements de concentration, et semblaient des affaires de familles. En 1911, la Société générale des tramways de Royan est présidée par L. Sarlin, assisté de A. et E. Filleux, de Royan, et de deux Parisiens ; en 1936, le conseil comprend un Parisien de plus et un Royanais de moins, mais la famille Sarlin tient encore la présidence.

\section{. Les matériels en service : de l'extinction de la cavalerie au triomphe de l'automotrice (tabl. 34)}

Les statistiques sur l'évolution du parc ne distinguent pas l'équipement des TVB de celui des TV; surtout, elles ne permettent pas de suivre l'électrification des réseaux. Face à un kilométrage exploité passant de 2153 à $3162 \mathrm{~km}$ (+ $46 \%$ ) entre 1901 et 1911, la cavalerie régresse de $77 \%$ (1 195 chevaux contre 5 165), le nombre de locomotives de $46 \%$ (253 à 136), tandis que celui des automotrices augmente de $50 \%$ (3 190 et 4 786) et celui des voitures de $26 \%$ (2 603 et 3 284). La définition retenue pour les automotrices inclut dans cette catégorie, en 1911, la totalité des tramways électriques presque généralisés en milieu urbain : « L'automotrice est une voiture comportant un moteur quelconque et un compartiment pour voyageurs ou messageries. Les voitures communiquant avec un conducteur aérien ou souterrain ont été classées dans cette catégorie.»

8- Président Petit et MM. Fréville, Lewandowski et Schweisguth.

9- Ministère des Travaux publics, Statistiques détaillées sur les CFIL et tramways an 31 décembre 1911 : départements et concessions, Paris, 1912. 
Tableau 34. Modes de traction sur divers réseaux

\begin{tabular}{|c|c|c|c|c|}
\hline Compagnies & $\mathrm{Km}$ & Che vaux & $\begin{array}{l}\text { Locomo- } \\
\text { tives }\end{array}$ & Autom otrices \\
\hline $\begin{array}{l}\text { Tramw ay de Dunkerque à Saint-Pol- } \\
\text { sur-Mer }\end{array}$ & 3 & 21 & & \\
\hline $\begin{array}{l}\text { Tramw ay de Lyon à Neuville-sur- } \\
\text { Saône }\end{array}$ & 16 & & 12 & \\
\hline Tramw ay de Royan & 15 & & 11 & \\
\hline Tramw ay de Bayonne à Biarritz (a) & 9 & & 6 & \\
\hline $\begin{array}{l}\text { Chemin de fer de Clermont-Ferrand } \\
\text { au sommet du Puy-de-Dôme }\end{array}$ & 15 & & 5 & \\
\hline \begin{tabular}{|l|} 
Compagnie de chemin de fer du \\
Var et du Gard : réseau de Toulon
\end{tabular} & 31 & 2 & & 54 \\
\hline Tramw ay de Boulogne-sur-Mer & 16 & 2 & & 27 \\
\hline Réseau de Toulouse & 91 & 45 & & 130 \\
\hline Réseau d'Amiens & 21 & 3 & & 40 \\
\hline $\begin{array}{l}\text { Compagnie générale française de } \\
\text { tramw ay : réseau du Havre }\end{array}$ & 62 & 2 & & 92 \\
\hline $\begin{array}{l}\text { Compagnie générale française de } \\
\text { tramw ay : réseau de Marseille }\end{array}$ & 166 & & 4 & 407 \\
\hline Tramw ay de Nantes & 45 & & 2 & 94 \\
\hline $\begin{array}{l}\text { Compagnie des tramw ays de Paris } \\
\text { et de la Seine }\end{array}$ & 217 & & 10 & 312 \\
\hline $\begin{array}{l}\text { Compagnie générale des omnibus } \\
\text { de Paris }\end{array}$ & 175 & 1061 & 8 & 366 \\
\hline
\end{tabular}

L'automotrice est le matériel caractéristique des tramways : pour les TV elle peut offrir deux classes aux voyageurs, elle peut tracter deux ou trois voitures, les baladeuses, en s'adaptant à la demande ; pour les TVB, elle offre les mêmes avantages, en outre, sur les lignes à faible trafic, elle assure le transport des voyageurs et des messageries sans demander la présence d'un second véhicule. L'évolution des modes de traction est le fait majeur pour les réseaux de TV comme de TVB.

En 1889, le tramway électrique circulait à Marseille, Lyon, Dijon, Angers, Rouen, Le Havre..., la province était en avance sur la capitale vouée aux chevaux ${ }^{10}$. Divers progrès techniques avaient été mis au point pour réduire les émissions de fumée (locomotive sans foyer, automotrices

10- Voir par ex. G. d'Avenal, «Les mécanismes de la vie moderne. Les moyens de transports urbains. Tramways. Bateaux et Métropolitain ", Revue des Deux-Mondes, $1^{\mathrm{er}}$ avril 1903, p. 583-609. 
Serpollet, Rowan, Purrey) « pour ne pas transformer la ville entière en une immense gare de chemin de fer $»^{11}$; d'autres procédés étaient utilisés : substitution des huiles lourdes au coke, moteur à air comprimé, chacun avait son application sur l'une ou l'autre des lignes des compagnies parisiennes ; ces énergies avaient en commun d'être onéreuses surtout pour le véhicule unique qu'était souvent le tramway. Le salut est venu de l'électricité, mais il fallut vaincre les oppositions de ceux qui se méfiaient des câbles électriques souterrains ou dans les caniveaux ${ }^{12}$ ou qui craignaient de voir les trolleys ${ }^{13}$ et le fil conducteur déparer les perspectives des avenues parisiennes. A ces modes de traction s'ajoutait la traction animale : la Compagnie générale des omnibus de Paris y était en 1911 encore largement fidèle ; elle y recourait pour $28 \%$ des kilomètres parcourus ${ }^{14}$ contre $2 \%$ aux locomotives et $70 \%$ aux automotrices; elle était aussi demeurée largement fidèle aux systèmes mécaniques. Les autres compagnies parisiennes avaient opté pour l'électricité, mais sous des formes diverses. Orienter, par le cahier des charges, les entreprises vers la recherche de nouveaux modes de traction (moteur à explosion alimenté en essence), conduire à l'uniformisation des méthodes employées en traction électrique assurant une compatibilité totale entre réseaux étaient des orientations étrangères aux forces alors en présence, qui se seraient inscrites dans la même logique que la volonté d'établir un réseau d'ensemble cohérent à l'échelle de Paris et de la Seine. On voit poindre seulement la politique, tellement plus longue à réaliser qu'à Berlin ou à Londres, tendant à confier à un opérateur unique, ou au moins à une autorité couvrant tous les opérateurs, l'organisation des transports parisiens.

Hors Paris, la traction animale est seule, avec 21 bêtes, à assurer la liaison de $3 \mathrm{~km}$ entre Dunkerque et Saint-Pol-sur-Mer. À Toulouse, la part du trafic assuré par des chevaux n'est pas isolée dans les statistiques. Les autres compagnies disposent d'une cavalerie insignifiante. Les locomotives sont seules en service sur quatre réseaux et utilisées parallèlement aux automotrices sur trois autres. En fait, les automotrices seules comme engins de traction répondent aux besoins de $77 \%$ des réseaux en exploitation. Leur nombre est fonction de la longueur du

11- Art. cité, p. 285.

12- Leur crainte majeure et irraisonnée avait pour motif les fers des chevaux.

13- Cette technique a triomphé ; elle équipait en $190390 \%$ des réseaux urbains électrifiés en service en Allemagne, Angleterre, Belgique, France.

14- La traction animale assurait $5978057 \mathrm{~km}$ parcourus, soit une moyenne quotidienne de $15,4 \mathrm{~km}$ par cheval. 
réseau et du trafic attendu : on en compte cinq sur la liaison d'Aubenas à Vals-les-Bains (8 km), 38 pour le réseau d'Orléans, 224 et 17 pour ceux de Bordeaux et Caen. Les automotrices peuvent tirer deux ou trois voitures ; le nombre de ces dernières est presque toujours inférieur à celui des automotrices. Sur certaines lignes courtes comme SaintChamond à Izieux aucune voiture n'est en service $(2 \mathrm{~km}$ et cinq automotrices). La situation se vérifie même sur des réseaux comme à Angers (22 automotrices pour $14 \mathrm{~km}$ ) et à Châlons-sur-Marne (six automotrices pour cinq $\mathrm{km})$.

La traction animale a un rôle encore plus faible sur les réseaux de TVB que sur ceux des TV : 22 chevaux assurent en exclusivité le service sur les six kilomètres séparant Épernay de Mareuil-sur-Ay ; chaque cheval parcourait une moyenne quotidienne de $18 \mathrm{~km}$, pour un total annuel de $147000 \mathrm{~km}$ sur la ligne ; deux autres compagnies entretenaient 20 et 23 chevaux face à 286 et 201 automotrices, la Compagnie générale parisienne de tramways et le Réseau urbain et suburbain de Lille ; le kilométrage assuré par la cavalerie n'apparait pas dans les statistiques. Sept compagnies n'utilisent que des locomotives sur de courtes lignes de 3 à $10 \mathrm{~km}$ à l'exception des $23 \mathrm{~km}$ des tramways de Savoie ; ces lignes unissent des gares aux localités voisines ou se trouvent dans des zones où se déplacent des touristes ${ }^{15}$. Sept compagnies partagent la traction entre locomotives et automotrices : trois et quatre entre Tours et Vouvray comme entre Gérardmer, Retournemer et La Schlucht ; une seule liaison est pourvue d'un parc important de locomotives, 35 et 37 automotrices entre Firminy, Saint-Étienne et Rive-de-Gier, sans doute la production locale de charbon a-t-elle orienté ce choix. Restent 23 compagnies qui ne disposent que d'automotrices; elles desservent aussi bien des réseaux urbains de quelques kilomètres que des lignes de 15 à $30 \mathrm{~km}$. Le nombre de voitures par rapport à l'effectif d'automotrices est commandé par les fréquences et les pointes de trafic sensibles, sur certaines lignes, aussi bien pour les promenades dominicales que pour les déplacements de travail ${ }^{16}$. Seulement 84 wagons spécialisés dans l'acheminement des messageries sont en service. 21 compagnies n'en font pas usage, confiant ce trafic aux automotrices ;

15- Gargan-Livry à Gargan-ville, Villers-le-Bel-gare à Villers-le-Bel-ville, Saint-Malo à Paramé et Rothéneuf, Etaples à Paris-plage, Société de Quend-plage (de la plage à Quend-gare), Tramway du Mont-Blanc (Le Fayet à l'Aiguille du Gouter).

16- Tramways de l'est parisien 57 voitures pour 282 automotrices, Réseau de Lille 103 et 201, Réseau de Caen 15 et 25, Moutiers à Brides-les-Bains 2 et 4, a contrario Marseille à Aix-en-Provence 18 et 10 . 
beaucoup se satisfont de un à trois wagons, par exemple sur les lignes de Bordeaux à Léognan ou de Poissy à Saint-Germain-en-Laye ; très rares sont les lignes dépassant cet effectif ${ }^{17}$.

Une synthèse du niveau d'équipement des réseaux de TV et de TVB peut être proposée en se fondant sur un indice parc/km exploité, mais en se limitant aux types de matériel suffisamment représentés pour donner un résultat significatif: les automotrices et les voitures (tabl. 35). Les compagnies parisiennes et provinciales de TV présentent des indices d'équipement pratiquement semblables; elles évoluent prioritairement en milieu urbain et dans les banlieues proches. Les réseaux de TVB sont moins bien dotés, notamment en voitures ; les automotrices suffisent à faire face à la demande de transport des voyageurs et des messageries.

Tableau 35. Parc de matériel et niveau d'équipement des lignes de TV et TVB (a)

\begin{tabular}{|c|c|c|c|c|c|c|}
\hline \multirow{3}{*}{ Matériel } & \multicolumn{2}{|c|}{ TVB (919km) } & \multicolumn{4}{|c|}{ TV (2 243 km) } \\
\hline & \multirow{2}{*}{ Nom bre } & \multirow{2}{*}{ Indice } & \multicolumn{2}{|c|}{ Paris-Seine $(520 \mathrm{~km})$} & \multicolumn{2}{|c|}{ Province (1723 km) } \\
\hline & & & Nombre & Indice & Nom bre & Indice \\
\hline Chevaux & 65 & & 1061 & & 70 & \\
\hline Locomotives & 78 & & 18 & & 40 & \\
\hline Autom otrices & 1152 & 1,25 & 879 & 1,7 & 2755 & 1,6 \\
\hline Voitures & 750 & 0,81 & 594 & 1,1 & 1940 & 1,1 \\
\hline Wagons & 84 (b) & & & & 9 (b) & \\
\hline
\end{tabular}

(a) On a fait figurer pour mémoire le parc des matériels dont le petit nombre excluait le calcul d'un indice significatif.

(b) $5 \mathrm{w}$ agons circulent sur le réseau urbain de Marseille, 4 entre Toulon et Hyères, ces lignes étant cependant classées TV.

\section{. Les personnels}

Une remarque préalable s'impose. La Compagnie générale des omnibus de Paris emploie 11276 personnes, soit $32 \%$ du total national des agents des compagnies de tramways et $72 \%$ de ceux des entreprises parisiennes; or cette entreprise ne fournit aucune indication sur la répartition de son personnel entre les services, interdisant certaines comparaisons. La raison est l'utilisation des mêmes personnels aussi bien pour les tramways que pour les omnibus à chevaux, qui ne relèvent pas de l'activité ferroviaire.

17- Cagnes à Menton six wagons, Boulogne-sur-Mer à Hardelot 8 , Firminy à SaintÉtienne et Rive-de-Gier 12. 
L'effectif national du secteur passe entre 1901 et 1911 de 28014 à 37436 actifs, la progression est d'un tiers, elle s'accélère après 1906, le taux moyen annuel est de 1,1\% avant cette date, de plus de $3 \%$ trois ans après ; parallèlement le réseau s'allongeait de $46 \%$. On peut esquisser une comparaison entre trois ensembles ; l'indice proposé est celui du nombre d'agents par kilomètre (tabl. 36).

Tableau 36. Part des effectifs affectés aux divers réseaux

\begin{tabular}{|l|c|c|c|c|c|}
\hline & Km & Effectif & Indice & $\begin{array}{c}\text { Part des } \\
\text { Km (\%) }\end{array}$ & $\begin{array}{c}\text { Part des } \\
\text { personnels (\%) }\end{array}$ \\
\hline TVB & 919 & 7034 & 7,6 & 29,5 & 18,7 \\
\hline TV Paris, Seine & 520 & 15507 & $29,8(a)$ & 16 & 41,4 \\
\hline TV province & 1723 & 14885 & 8,6 & 54,5 & 39,9 \\
\hline
\end{tabular}

(a) Cet indice est anormalement élevé par suite des résultats de la Compagnie générale des omnibus de Paris, 64,4 . Si on élimine cette compagnie, les données pour les autres entreprises parisiennes sont de 4231 salariés pour $345 \mathrm{~km}$, soit un indice de 12,2. Le volume du trafic pèse fortement sur les effectifs nécessaires pour y faire face, donc sur l'indice.

Avant d'aborder des exemples, quelques évidences gagnent peutêtre à être rappelées.

Le kilométrage à entretenir est réduit ; la lenteur des convois limite l'usure des rails, soumis cependant au piétinement des chevaux et au passage des roues des charrois. À l'exception de rares entreprises disposant de services administratifs étoffés (325 agents pour la Compagnie des tramways de Paris et de la Seine, 90 pour le réseau de Marseille de la Compagnie générale française de tramways...), les effectifs sont souvent inférieurs à cinq, les bâtiments pour voyageurs se limitent à quelques kiosques et abris sommaires à quelques arrêts, seuls restent les bâtiments de maintenance des dépôts. Tout concourt à la faiblesse des effectifs de la division Voie et Bâtiments qui dépasse rarement $10 \%$ de la main-d'œuvre employée et s'abaisse souvent autour de $6 \%$ (tabl. 37). 
Tableau 37. Répartition des emplois dans les réseaux de tramways pour voyageurs seulement (TV)

\begin{tabular}{|c|c|c|c|c|c|c|c|c|}
\hline Compagnies & $\mathrm{Km}$ & & $\begin{array}{l}\text { Total des } \\
\text { emplois }\end{array}$ & $\begin{array}{l}\text { Indice } \\
\text { (a) }\end{array}$ & A (b) & $E(c)$ & MT (d) & VB (e) \\
\hline \multirow{2}{*}{$\begin{array}{l}\text { Compagnie des tramw ays } \\
\text { de Paris et de la Seine }\end{array}$} & 217 & Nombre & 2759 & 12,7 & 323 & 847 & 1344 & 245 \\
\hline & & $\%$ & & & 11,7 & 30,6 & 48,7 & 9 \\
\hline \multirow{2}{*}{ Chemins de fer nogentais } & 55 & Nombre & 712 & 12,9 & 34 & 276 & 308 & 94 \\
\hline & & $\%$ & & & 4,7 & 38,8 & 43,3 & 13,2 \\
\hline \multirow{2}{*}{$\begin{array}{l}\text { Compagnie électrique des } \\
\text { tramw ays de la rive } \\
\text { gauche de Paris }\end{array}$} & 34 & Nombre & 345 & 10,1 & 20 & 127 & 166 & 32 \\
\hline & & $\%$ & & & 5,8 & 36,8 & 48,2 & 9,2 \\
\hline \multirow{2}{*}{ Réseau d'Angers } & 14 & Nombre & 89 & 6,3 & 5 & 40 & 35 & 9 \\
\hline & & $\%$ & & & 5,6 & 44,9 & 39,3 & 10,2 \\
\hline \multirow{2}{*}{ Réseau de Nîmes } & 15 & Nombre & 104 & 6,9 & 1 & 5 & 71 & 27 \\
\hline & & $\%$ & & & 1 & 4,8 & 68,2 & 26 \\
\hline \multirow{2}{*}{ Réseau de Dijon } & 16 & Nombre & 108 & 6,7 & 4 & 85 & 14 & 5 \\
\hline & & $\%$ & & & 3,7 & 78,7 & 13 & 4,6 \\
\hline \multirow{2}{*}{$\begin{array}{l}\text { Réseau de Châlons-sur- } \\
\text { Marne }\end{array}$} & 5 & Nombre & 19 & 3,8 & 0 & 15 & 3 & 1 \\
\hline & & $\%$ & & & 0 & 79 & 15,8 & 5,2 \\
\hline \multirow{2}{*}{ Réseau de Belfort } & 5 & Nombre & 40 & 8 & 3 & 10 & 14 & 13 \\
\hline & & $\%$ & & & 7,5 & 25 & 35 & 32,5 \\
\hline $\begin{array}{l}\text { Société des tramw ays de } \\
\text { Royan : } \quad \text { hors saison }\end{array}$ & 15 & Nombre & 24 & 1,6 & 3 & 3 & 18 & 0 \\
\hline saison & 15 & Nombre & 57 & 3,8 & 3 & 18 & 29 & 7 \\
\hline hors saison & & $\%$ & & & 12,5 & 12,5 & 75 & 0 \\
\hline saison & & $\%$ & & & 5,2 & 31,5 & 50,8 & 12,5 \\
\hline \multicolumn{9}{|c|}{ Compagnie générale française de tramways } \\
\hline Réseau de Marseille & 166 & Nombre & 2582 & 15,5 & 90 & 1680 & 752 & 80 \\
\hline Réseau d'Orléans & 27 & Nombre & 155 & 5,7 & 5 & 107 & 33 & 10 \\
\hline Réseau de Nancy & 34 & Nombre & 351 & 10,3 & 11 & 265 & 53 & 27 \\
\hline Réseau du Havre & 62 & Nombre & 543 & 8,7 & 17 & 325 & 85 & 116 \\
\hline Réseau de Marseille & & $\%$ & & & 3,5 & 65,1 & 28,4 & 3 \\
\hline Réseau d'Orléans & & $\%$ & & & 3,2 & 69,1 & 21,2 & 6,4 \\
\hline Réseau de Nancy & & $\%$ & & & 3,2 & 75,5 & 15,1 & 6,2 \\
\hline Réseau du Havre & & $\%$ & & & 3,7 & 59,8 & 15,5 & 21 \\
\hline
\end{tabular}

(a) Nombre d'agents au kilomètre.

Nombre d'emplois et pourcentage pour chaque service : (b) Services administratifs - (c) Service de l'Exploitation - (d) Service Matériel et Traction - (e) Service Voie et Bâtiments 
Les divisions Exploitation et Matériel et Traction réunissent le gros des troupes. Le fonctionnement du système l'exigeait. Le tramway devait en effet présenter deux qualités essentielles, la fréquence et l'amplitude des services. D'abord la fréquence variable pour s'adapter aux déplacements urbains. Si, en début et fin de services, les intervalles pouvaient être d'une demi-heure ou plus, ils devaient dans la journée s'abaisser à 10 minutes ou moins aux heures de plus forte fréquentation. La possibilité de modeler les fréquences était limitée par les croisements en nombre restreint à mesure qu'on s'éloignait du centre. L'amplitude du service était fonction de la dimension des villes et de leur composition sociologique. N’oublions pas la durée de la journée de travail qui commençait dans les usines entre $6 \mathrm{~h}$ et $6 \mathrm{~h} 30$. Dans les grandes villes, les circulations avaient lieu en général de $5 \mathrm{~h}$ à $21 \mathrm{~h}$ ou $22 \mathrm{~h}$, dans les autres de $6 \mathrm{~h}$ ou $7 \mathrm{~h}$ à $20 \mathrm{~h}$ ou $21 \mathrm{~h}$; la présence d'une population ouvrière tendait à avancer l'heure de départ des premiers tramways. Dans de nombreuses villes, les compagnies assuraient le service des derniers trains, de même qu'un service à la sortie du théâtre.

Un personnel nombreux était nécessaire pour faire face à ces obligations. Même avec une législation sociale moins élaborée qu'elle ne le deviendra après 1920, les mêmes agents, notamment les conducteurs, ne pouvaient être astreints à travailler plus de 13 ou plus de 16 heures par jour suivant la compagnie. Conducteurs, receveurs dans l'automotrice, éventuellement dans les remorques, contrôleurs, aiguilleurs à quelques carrefours stratégiques du réseau ${ }^{18}$, personnel d'entretien du matériel roulant et des fils conducteurs étaient indispensables en roulement.

Quelques exemples montrent qu'il n'y a pas de règle dans la place relative tenue dans l'emploi par les divisions Exploitation et Matériel et Traction. La première domine avec des taux élevés dans les réseaux de la Compagnie générale française de tramways (60 à $75 \%$ ) et dans celui de Châlons-sur-Marne; la seconde l'emporte pour les trois compagnies parisiennes citées, mais sans jamais atteindre $50 \%$; un certain équilibre y semble réalisé. Trois villes de poids démographiques voisins, dotées de réseaux de longueurs comparables offrent des résultats totalement opposés ${ }^{19}$. Ces observations conduisent à se demander comment des réseaux pouvaient fonctionner avec de telles différences dans la gestion de leur personnel ou s'il s'agit d'une faiblesse dans l'élaboration des statistiques, notamment en matière de définition des emplois. Enfin

18- Certaines aiguilles étaient manipulées par les receveurs, jusqu'à ce qu'une commande à distance à l'approche d'une rame ait été mise au point.

19- Dijon 77000 habitants ; Nîmes 80000 ; Angers 83000. 
des réseaux doivent s'adapter aux variations saisonnières du trafic liées aux activités touristiques. La Société des tramways de Royan est un exemple avec l'importance des emplois saisonniers. Le réseau de Lourdes offre une situation identique, mais ici ce sont les pèlerins qui créent le mouvement : les pèlerinages régionaux organisés se succédaient de mai à octobre. Dans une France encore très rurale, les pèlerinages d'arrière-saison, après les grands travaux agricoles, étaient très fréquentés.

Le nombre d'agents $/ \mathrm{km}$ augmente avec le volume de population desservie, mais le rapport n'est pas direct ; il est plus élevé sur les réseaux urbains de TV, que sur les réseaux suburbains de TVB ; à Angers 6,3 et 4,4 : cela s'explique par une fréquence plus faible, suffisante pour répondre à la demande. Le personnel des tramways se caractérise par une hiérarchie beaucoup moins élaborée que sur les réseaux de CFIL et de TVM ; les agents sédentaires du service de l'exploitation sont très peu nombreux, voire absents.

Ces réseaux, qui répondent à des besoins spécifiques où la desserte des milieux urbains est l'objectif, disposent de moyens adaptés à la satisfaction de cette demande, tant pour les matériels que pour les personnels.

Les TV parcourant prioritairement les centres des villes et les quartiers proches desservent des zones à forte densité. Les migrations quotidiennes et saisonnières de travail et de scolarité assurent un fond de clientèle potentielle élevé, comparé à celui existant le long des lignes rurales. Les recettes kilométriques sont importantes. Cette situation favorable ne doit pas faire oublier le manque de perspectives d'avenir dans la gestion de bien des réseaux : négligences dans l'entretien et le renouvellement des voies et du matériel et, surtout, manque d'efforts pour adapter le réseau à la croissance de la ville. Les témoins des années trente voient encore le tramway bruyant avec ses remorques, brinquebalant, se faufilant dans les rues étroites ; ils se souviennent des attentes pour les croisements, des arrêts dus aux trolleys qui sautaient.

Même si les TV et les TVB ont été relativement privilégiés, les difficultés financières ne les ont pas épargnés et ont contribué à précipiter la conversion vers la technique routière. Même en ville, le chemin de fer, au sens littéral du terme, était condamné, avant de connaittre une faveur nouvelle.

\section{La situation financière des entreprises de TV et de TVB en 1911}

Les résultats d'exploitation sont en moyenne meilleurs que ceux des autres VFIL : coefficients d'exploitation plus favorables, souvent inférieurs à $70 \%$, petit nombre de lignes déficitaires (tabl. 38 et 39). 


\section{. Les TVB}

37 réseaux de TVB fonctionnent en $1911 ; 21$ ont moins de $10 \mathrm{~km}$, cinq dépassent 50 , desservant en général des villes et leur périphérie. Bien des lignes de TVB dépassent en distance de nombreuses lignes de TVM, ce type de liaisons bénéficie de coefficients d'exploitation compris entre 60 et $80 \%$. Les courtes lignes correspondent à des réseaux urbains dont la prospérité dépend du potentiel de clientèle offert par la ville ou à des liaisons entre une localité et une gare; des lignes de ce type sont parfois déficitaires, ce qui est aussi le cas de la liaison de Nancy à Dombasle et à Pont-à-Mousson $(29 \mathrm{~km})$; là, le déficit est de $-49738 \mathrm{~F}$, inférieur à ceux enregistrés par certaines lignes de CFIL et TVM (tabl. 38).

Tableau 38. Dépenses d'établisements, produits nets, coefficients d'exploitation d'un échantillon de lignes TVB

\begin{tabular}{|l|c|r|r|r|}
\hline \multicolumn{1}{|c|}{ Com pagnies } & Km & $\begin{array}{r}\text { Dépenses } \\
\text { d'établisse- } \\
\text {-ment (en F) }\end{array}$ & $\begin{array}{c}\text { Produits } \\
\text { nets (en F) }\end{array}$ & D/R (\%) \\
\hline Réseaux urbain et suburbain de Lille & 127 & 312071 & 1956994 & 61,2 \\
\hline $\begin{array}{l}\text { Compagnie générale parisienne de } \\
\text { tramw ays }\end{array}$ & 90 & 877945 & 1936926 & 80,6 \\
\hline Tramw ays de l'est parisien & 184 & 397033 & 1755552 & 74,1 \\
\hline Tramw ays de Nice et du littoral & 81 & 431441 & 1183076 & 64,5 \\
\hline $\begin{array}{l}\text { Chemin de fer voie étroite de Saint- } \\
\text { Etienne à Firminy, Rive-de-Gier et } \\
\text { extensions }\end{array}$ & 64 & 169723 & 628851 & 68,7 \\
\hline Réseau de Cannes et banlieue & 25 & 119103 & 220201 & 57,8 \\
\hline Réseau suburbain d'Angers & 16 & 168004 & 116451 & 56,2 \\
\hline Réseau de Fontainebleau & 6 & 184260 & 88595 & 53,5 \\
\hline Versailles à Saint-Cyr-l'Ecole (a) & 4 & 167162 & 40135 & 53,7 \\
\hline Tramw ays électriques de Blois & 7 & 100384 & 7048 & 91,3 \\
\hline Epernay à Mareuil-sur-Ay & 6 & 47185 & 6707 & 87,5 \\
\hline Moutiers à Brides-les-Bains & 6 & 98464 & 1593 & 95,5 \\
\hline
\end{tabular}

(a) La Société versaillaise de tramw ays électriques et distribution d'énergie exploite aussi le réseau urbain de TV.

Le coût moyen des dépenses d'établissements des TVB est au $\mathrm{km}$ cinq fois supérieur à celui d'une ligne de TVM, légèrement supérieur à celui d'un TV de province, très inférieur à celui d'un TV de Paris (tabl. 39). Les aménagements de la voie publique en ville ont un coût élevé et, sauf exception, les TVB sont tracés en zone urbaine ou en voie 
d'urbanisation, rares sont les parcours en milieu rural. Beaucoup de TVB sont à traction électrique ; que l'alimentation soit aérienne ou souterraine, les investissements correspondant sont très lourds. Le tramway est un véhicule cher ; l'acquisition du matériel grève les dépenses d'établissement. Le TVB acheminant les messageries, quelques points d'arrêt doivent être équipés pour la livraison et la réception de marchandises. Malgré ces contraintes, presque toutes les compagnies exploitant un TVB ont fait face à $100 \%$ des dépenses d'établissement, seulement trois d'entre elles n'ont donné qu'entre 96 et $99,5 \%{ }^{20}$. Deux cas font exception : la ligne de Brest au Conquet a reçu des aides publiques pour $22 \%$ du montant, tandis que le département de la Savoie finançait seul la ligne de Moutiers à Brides-les-Bains.

\section{. Les TV}

Un constat de même nature peut être fait pour les lignes de TV (tabl. 39). L'aide publique intervient pour $11 \%$ pour la ligne de Clermont-Ferrand au sommet du Puy de Dôme, pour un pourcentage très faible pour quelques villes ${ }^{21}$.

On compte 78 lignes ou réseaux de TV; dix dépassent $50 \mathrm{~km}$, mais 28 comptent de 2 à $10 \mathrm{~km}$. Deux lignes seulement présentent un déficit d'exploitation. Le kilométrage exploité n'influence pas la valeur du coefficient d'exploitation, identique pour les réseaux de Lyon $(238 \mathrm{~km})$ et de Saint-Chamond $(2 \mathrm{~km})$. Les résultats d'exploitation et les dépenses d'établissement enregistrés sur un certain nombre de lignes de caractéristiques diverses par leur longueur et par la nature des villes desservies conduisent à une série de constatations et d'interrogations. Soulignons l'originalité de Paris et de la Seine qui se singularisent par la concurrence entre sept compagnies de TV et deux de TVB; les grandes villes de province échappent presque toutes à cette situation (tabl. 38).

Les données suggèrent un parallélisme entre la diminution de la longueur d'un réseau exploité et celles du produit net et des dépenses d'établissement; chaque paramètre évolue à son propre rythme et cette évolution souffre des exceptions.

Le produit net dépend en partie du volume des recettes ; un réseau urbain de 15 à $20 \mathrm{~km}$, ou beaucoup plus, transporte plus de voyageurs qu'une ligne de 2 à $5 \mathrm{~km}$; les recettes et donc le produit net traduisent cette réalité. Les recettes des compagnies desservant Paris et les grandes villes de province se chiffrent en millions, celles des autres villes en dizaines, plus fréquemment en centaines de milliers de francs.

20- TVB de Rennes, Fontainebleau, Grenoble.

21- Belfort, Bordeaux, Nantes, Lyon, Tours, Royan. 
Tableau 39. Dépenses d'établissement, produits nets et coefficients d'exploitation d'un échantillon de lignes de TV

\begin{tabular}{|l|c|c|c|c|}
\hline \multicolumn{1}{|c|}{ Exploitation } & Km & $\begin{array}{c}\text { Dépenses } \\
\text { d'établisse- } \\
\text { ment F/Km }\end{array}$ & $\begin{array}{c}\text { D/R } \\
\text { (\%) }\end{array}$ & $\begin{array}{c}\text { Produits } \\
\text { nets (F) }\end{array}$ \\
\hline Omnibus des tramw ays de Lyon & 238 & 259178 & 62 & 4172559 \\
\hline Réseau de Marseille & 166 & 469202 & 69 & 3288534 \\
\hline Générale des omnibus de Paris & 175 & 560721 & 89,1 & 2121553 \\
\hline Réseau de Bordeaux & 101 & 497873 & 65,6 & 2065577 \\
\hline Chemin de fer nogentais & 55 & 371614 & 65,4 & 1320606 \\
\hline Réseau de Rouen & 69 & 307364 & 57,5 & 1210514 \\
\hline Réseau de Toulouse & 91 & 184946 & 62,6 & 929013 \\
\hline Réseau du Havre & 62 & 291042 & 63,4 & 866775 \\
\hline $\begin{array}{l}\text { Tramw ays de Paris et du } \\
\text { département de la Seine }\end{array}$ & 217 & 267173 & 93,9 & 635772 \\
\hline $\begin{array}{l}\text { Réseaux urbains et suburbains } \\
\text { de Roubaix et Tourcoing (a) }\end{array}$ & 54 & 194588 & 72,6 & 572238 \\
\hline Réseau de Reims & 24 & 366780 & 66,2 & 350107 \\
\hline Réseau de Boulogne-sur-Mer & 16 & 212563 & 59,2 & 159885 \\
\hline Réseau de Brest & 17 & 211510 & 64 & 159463 \\
\hline Réseau de Dijon & 14 & 217879 & 61,7 & 159340 \\
\hline Réseau de Belfort & 5 & 115000 & 64,7 & 61694 \\
\hline Réseau de Bourges & 9 & 177250 & 72,8 & 56494 \\
\hline Réseau de Poitiers & 2 & 225423 & 85,9 & 23428 \\
\hline Réseau d'Armentières & 5 & 193314 & 76,7 & 21903 \\
\hline Réseau de Saint-Chamond & 231775 & 62,6 & 20845 \\
\hline Réseau de Châlons-sur-Marne & 5 & 190214 & 72 & 20409 \\
\hline Réseau de Melun & 259000 & 85,4 & 10000 \\
\hline Réseau d'Elbeuf & 282320 & 100,9 & -1928 \\
\hline Wimereux à l'Hippodrome & 218,4 & -8467 \\
\hline (a) Couvre les deux villes ; il est indépendant du TVB urbain et suburbain de Lille \\
\hline poumble de l'agglomération & 231 km. & & & \\
\hline
\end{tabular}


Mais, entre longueur et produit net, la proportion est loin d'être parfaitement respectée. Le plus long réseau parisien (Tramways de Paris et du département de la Seine) présente un produit net inférieur à celui d'autres réseaux parisiens ou de grandes villes de province ; ses recettes ne sont que de $53 \%$ de celles de la Compagnie générale des omnibus de Paris et son produit net de $30 \%$. L'explication tient sans doute à la nature des espaces urbains desservis par chacune de ces compagnies ; si on se réfère à l'article de la Revue des Deux-Mondes déjà évoqué, on y voit la Compagnie générale des omnibus de Paris reléguer ses concurrentes sur des lignes de banlieue peu productives ${ }^{22}$. De même, au bas du tableau, le réseau de $5 \mathrm{~km}$ de Belfort a un produit net supérieur à celui enregistré sur des réseaux de 6 à $9 \mathrm{~km}$, et le réseau déficitaire d'Elbeuf ne se situe pas parmi les plus courts (tabl. 39). La baisse des dépenses d'établissement parallèlement à celle du kilométrage exploité est une tendance certaine, mais irrégulière. Sur les réseaux de TV, rappelons qu'en 1911 l'électrification est pratiquement généralisée ; son coût pèse sur les dépenses d'établissement.

Un réseau de quelques kilomètres compte souvent une seule, voire deux lignes ; les sections communes à plusieurs lignes n'existent pas ou constituent une très faible part du réseau ; les fréquences sont généralement moindres que dans les grandes villes. Ces conditions favorisent la construction à voie unique, parce qu'il suffit de quelques points de croisement pour permettre la rotation du matériel, dont le parc est peu étoffé. Au contraire, dans les grandes villes, au moins dans la partie centrale des réseaux, la voie double est indispensable. La durée des parcours limitant la rotation du matériel, le volume de la clientèle et le développement des pointes de trafic imposent de disposer d'un parc de matériel fourni, composé d'automotrices et de remorques, moins chères à l'achat et permettant d'adapter l'offre à la demande.

Les dépenses d'établissement dépendent aussi des facilités inégales offertes par la voirie : largeur des artères, difficultés dans les virages, croisements de lignes. Le site n'est pas sans influence : les fortes rampes à vaincre pour unir une gare au cœur de la ville traditionnelle n'augmentent-elles pas les dépenses d'établissement et, ultérieurement, d'exploitation? Les collines ponctuant le site d'une ville, comme à Marseille, imposent des détours pour les contourner ou des parcours sinueux par des artères inadaptées, héritées des chemins du passé, pour les gravir. Les sources d'augmentation des dépenses ne manquent pas.

22- G. d'Avenal, art. cité, 1903. 
Mais que de questions viennent à l'esprit ! Pourquoi, entre les deux premières entreprises parisiennes, les dépenses d'établissement au $\mathrm{km}$ varient-elles du simple à plus du double ? Pourquoi Bordeaux avec ses boulevards, les barrières ceinturant la ville, ou ses larges dégagements dus aux intendants du XviII ${ }^{\mathrm{e}}$ siècle - voirie et site relativement faciles - compte-t-elle des dépenses supérieures à Marseille et presque doubles de celles de Lyon, villes qui n’offrent pas les mêmes facilités ? Surprise aussi de trouver la même dépense au kilomètre à Lyon $(238 \mathrm{~km})$ et à Melun $(2 \mathrm{~km})$. Au contraire, dans certains cas, on enregistre une relative homogénéité des données, comme à Boulogne-sur-Mer, Brest et Dijon : égalité des produits nets et résultats proches pour les autres paramètres.

Les coefficients d'exploitation traduisant une rentabilité médiocre des réseaux parisiens principaux ou de ceux de Poitiers et d'Elbeuf s'opposent aux multiples situations saines observées sur les autres exploitations où les coefficients s'échelonnent entre 57 et $73 \%$.

Une interprétation complète et correcte de l'ensemble de ces données exigerait une connaissance au cas par cas de l'environnement politique et des impératifs financiers qui ont entouré l'octroi des concessions. Il conviendrait encore d'analyser les cahiers des charges et les mesures prises ou non pour développer les réseaux (nouvelles concessions, avenants) et les adapter à l'extension des villes dans l'espace.

\section{Conclusion}

Nous avons dans cette deuxième partie accordé aux entreprises et à leurs problèmes financiers une attention particulière. Les recherches doivent conduire à la connaissance des acteurs, et en premier lieu les concédants et les concessionnaires. Les débats dans les conseils généraux et municipaux et ceux dans l'opinion publique, évoqués entre autres à travers la presse, ont toujours été minutieusement étudiés par les chercheurs. L'entreprise concessionnaire a été généralement prise en compte, non pour elle-même, mais comme chargée d'exécuter les décisions des élus : respecterait-elle le tracé ou négocierait-elle d'autres cheminements, respecterait-elle le cahier des charges, réaliserait-elle rapidement le projet et la mise en service de la ligne, les prévisions de trafic étaient-elles réalistes, l'entreprise solliciterait-elle les finances départementales au-delà des engagements initiaux... ? Telles sont quelques-uns des thèmes principalement abordés dans les études de lignes ou de réseaux.

Or les entreprises sont diverses. Derrière l'entité juridique, il y a les hommes et les capitaux. 
Les fondateurs des entreprises n'appartiennent pas tous au même milieu. Connaitre leur origine sociale, leur implantation géographique, les liens qui les unissent et, à travers eux et en fonction des responsabilités qu'ils exercent, les liens tissés entre les entreprises, telles sont les connaissances qui éclairent la réalité du monde des chemins de fer secondaires.

La situation financière d'une entreprise est précisée dans le bilan annuel intégrant les données financières et les résultats d'exploitation. Nous avons insisté sur ces derniers, nous l'avons souligné, car ils sont l'expression du rôle de l'entreprise comme transporteur de voyageurs et de marchandises. Ces résultats indiquent les lignes déficitaires et les lignes bénéficiaires ; ils sont commandés en partie par le volume des recettes tirées du trafic réalisé. Les compagnies ont intérêt à le faire progresser comme le montrent les références relevées dans les procèsverbaux des assemblées générales des actionnaires de la SGCFE.

Des résultats d'exploitation favorables ne permettent pas d'appréhender la santé financière d'une entreprise. Il convient de prendre en compte la formation du capital en actions et en obligations, les modifications intervenues depuis l'origine dans la constitution du capital. Il faut aussi relever la part pour les actions et pour les obligations des capitaux amortis et à amortir, ainsi que le montant des intérêts à verser. Ces données doivent de préférence être étudiées pour plusieurs années.

Le champ des recherches dans ces domaines ouvre de larges perspectives avec la nécessité de procéder à des analyses comparatives entre des entreprises de divers types; les archives départementales doivent détenir pour certaines d'entre elles les éléments nécessaires, notamment les procès-verbaux des assemblées générales. Ces recherches prendront toute leur signification si, au-delà de la sécheresse statistique, elles s'inscrivent dans la problématique si diversifiée qui entoure les travaux sur les chemins de fer secondaires. 


\section{Annexe 1}

\section{Une entreprise à réseau unique}

Source : Annuaire des chemins de fer, des tramways et des services publics automobiles (Annuaire Marchal), 1936

\section{POITIERS}

\section{Compagnie des Tramways de Poitiers.}

Socièté anonyme, constituèe le 12 décembre 1896, pour 75 ans.

Siège social : rue de la Bourse, 8, Lyon.

Siège administratif : rue d'Athenes, 20, Paris (9•). Tèl.: Trinite 37-46, 37-45, 37-46. - Télèg. : Omnitram-Paris.

Direction et usıne : rue de la Souche, 4 , Poitiers. Tel. : 1-63 et 1-64.

DOCUMENTATION OFFICIELLE

Statuts déposés chez M/ Bodin, notaire à Poitiers, le 28 novembre 1896.

Durée de la concession : Jusqu'en 1946

Année sociale : 1*r janvier au 31 décembre.

Assemblée générale : Avant fin juin, composée des actionnaires propriêtaires de 25 actions. ACTES DE CONCESSION

Décret du 20 avril 1899 (J. O. 26 avril $1899-$ B. des L. 2125).

- $\quad 15$ mai 1903.

- $\quad 11$ avril 1920 (J. O. 15 avril 1920). Classement tramways urbains.

22 juin 1929 (J. O. du 28 juin 1929). Fixant le nouveau régime d'Exploitation.

- 13 février 1931 (J. O. du 23-24 février 1931). Relèvement des tarifs.

30 avril 1933 (J., O. du 6 mai 1933 ). Modification du régime d'exploitation.

CONSEIL. D'ADMINISTRATION

5 à 13 membres nommés pour 6 ans, propriétaires de 25 actions cnacun.

MI.

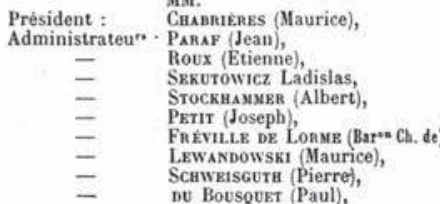

rue Paradis, 52,
rue de Miromesnil, 86,
avenue Mozart, 15 ,
r. des Belles-Feuilles, $48 \mathrm{bi}$,
avenue Mozart, 13,
rue de Pétrograd, 41,
rue Cassette, 12,
rue d'Assas, 74,
rue de Varenne, 55,
av, de Versailles, 130,

Marseille.

av. de Versailles, 130 ,

Paris $\left(8^{\circ}\right)$

- (16\%).

- $(16 *)$

$=(8 \cdot)$ Gut.14-7.

- Littré 76-49.

- 6*) Littré38-06.

COMMISSAIRES DES COMPTES

MM. Marchand (Raphaël), rue Lauriston, 104, Paris (15*).

Roux (Pierre), place Saint-Michel, 6, - (5*).

DIRECTEUR DE L'EXPLOITATION

M. Honoraz, rue de la Souche, 4, Poitiers. Tèl. : 1-63 et 1-54.

ORGANISATION FINANCIÉRE

Services financiers : Au siège administratif, rue d'Athènes, 20, Paris (9*).

Capital-Actions : 10.000 actions de $100 \mathrm{fr}$., soit 1.000 .000 de francs.

Répartition des bénéfices : $1^{\circ} 5 \%$ à la réserve légale jusqu'á ce qu'elle ait atteint 1/10 du capital social. 9* - Intérét de $5 \%$ aux actions.

$3^{*}$ - Prélèvements pour fonds d'amortissement du capital-actions ou réserve facultative.

L'excédent rèparti : $15 \%$ au Conseil d'administration, $85 \%$ aux actionnaires.'

\begin{tabular}{|c|c|c|c|}
\hline \multicolumn{4}{|c|}{$\begin{array}{l}\text { EXERCICE } 1934 \\
\text { Bilan. }\end{array}$} \\
\hline \multicolumn{2}{|l|}{$\begin{array}{l}\text { Actif. } \\
\text { Premier établissement .............. }\end{array}$} & \multicolumn{2}{|l|}{ Passif. } \\
\hline Premier établissement ............. & $951.778,34$ & Capital-Actions ................ & 1.000 .000$, \\
\hline utionnement................. & $14.411,19$ & Rèserve légale................... & $25.914,64$ \\
\hline Magasin ....................... & $108,300,09$ & Amortissements... ............ & $179.410,90$ \\
\hline Débiteurs divers................. & $43.405,26$ & Ville de Poitiers..$\ldots \ldots \ldots \ldots \ldots \ldots$ & $7.758,16$ \\
\hline Disponibilités...................... & $211,503,16$ & Crediteurs divers................. & $127.840,64$ \\
\hline Compte de gestion ville de Puitiers. & $87.513,80$ & $\begin{array}{l}\text { Reliquats des exercices anterieurs... } \\
\text { Bènéfices de l'exercice............... }\end{array}$ & $\begin{array}{r}611,39 \\
43,565,57\end{array}$ \\
\hline & & Fonds de renouvellement............ & $31.831,12$ \\
\hline & $1.616 .911,72$ & & $1.416 .911,72$ \\
\hline
\end{tabular}




\section{Profits et Pertes.}

Débit.

Excédent des dépenses du centre sur le

maximum contractuel..............

Amortissement

\begin{tabular}{r|}
10600,56 \\
$35.000,, 8$ \\
$43.545,57$ \\
\hline $89.146,13$ \\
\hline
\end{tabular}

Crèdit.

Rèmunération de la Ci- Service Autobus 13.397,22 Rémunération de la Ci-Service Tramways 72.571, 21 intèrêts et escomptes............... $3.177,70$

Répartition beneficiaire.

Réserve légale. Intéret au capital.

Report à nouveau.

\section{CONTROLE ADMINISTRATIF}

MM. Preduugarav, Ingènieur en Chef des Ponts et Chaussées, boul. de Verdun, 13, Poitiers. LANCrLisa, Ingenieur des T. P. E., boul. de Verdun, 13, -

\section{ECCCMENTATION TECHNIQLE}

Longueur du réseau : $6 \mathrm{~km}$.

Largeur de la voie: $1 \mathrm{~m}$.

Type de rail à ornière : 36 et $43 \mathrm{~kg}$.

Altitude minimum : $47 \mathrm{~m} .85$.

Altitude maximum : $117 \mathrm{~m} .97$.

Rayon minimum des courbes: $20 \mathrm{~m}$.

Traction electrique, prise de courant par archet.

PARC DU MATÉRIEL ROULANT

Automotrices de $7 \mathrm{~T}, 5$ i 26 places : 13 .

Autobus: 5 .

Remorques à 40 places ; 4 . 


\section{Annexe 2}

\section{Une entreprise fondée en 1875}

Source : Annuaire des chemins de fer et des tramways, 1927

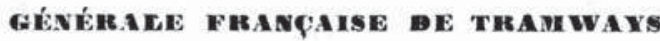

\section{Compagnie Générale FRANÇAISE de Tramways.}

Socielte anonyme, constituée le 17 décembrc 1875 , pour 115 ans.

Sizge social et Direction: 3, rue Moncey, Paris (9*). - Tél. : Gutenberg 16-60. Richelieu 95-49 Télègr.: Francotram-Paris.

\section{DOCOMENTATIOX OFFICIELLE}

Slatuts déposés chez M- PÉrard, notaire à Paris, le 8 décembre 1875, modifiés par les Assemblées générales des 6 avril 1891, 6 septembre 1896,23 janvier 1897,30 avril 1898,30 avril 1900, 17 avril 1901 5 mai 1905,15 mai 1907,27 juin et 3 octobre 1920,26 mai 1926.

Réseaux concédes :

Tramways du Havre.

$$
\begin{aligned}
& \text { de Marseille............. } 55 \mathrm{~km} \text {. } 426 \\
& \text { - de Nancy. } \\
& \text { - d'Orléans } \\
& 76-786 \\
& 334 \mathrm{~km} .684
\end{aligned}
$$

\section{Affaires domiciliées:}

Tramways de Saint-Quentin...... $9 \quad 9 \quad 769$

- de Toulon.............. 75 - 211

- de Tunis.............. $105-910$

$198 \mathrm{~km} .890$

Année sociale: 1 or janvier au 31 décembre.

Assembiée générale : En mars ou avril, composée des actionnaires propriétairos de 10 actions.

CONSEIL D ADMINISTRUTION

5 à 15 membres, nommés pour 6 ans, propriétaires de 50 actions chacun.

Prést honoraire : M. Rostand (Jules), 45, rue de Courcelles, Paris (8*) Tèl : Elysẻes 10-97.

Président :

M. PAvir (Georges), 3, rue Moncey, Paris (9॰). Tél, : Gutenberg 16-60.

Vice-Président: M. Guanx (Louis), 13, rue Daru, Paris (8*).

Adm, délégué : M. Borlue (Léon), 3 rue Thèodule-Ribot, Paris (17*

Administrateur : M. Axcion vax Por (baron Alfred), place Eunile-Dupont, à Liége (Belgique).

- M. Collignon (Auguste),

M. Collignon (Auguste),

M. Goort du Roslan (Louis). 1, rue Boccador, Paris (8*). Tél. : Elysées : 77-84.

9. Guiffart, 45 , rue Emile-Menier, Paris $\left(16^{\circ}\right)$.

M. Monmereué (ć), 19, rue Descamps (16*). Tél. : Passy 31-55.

M. Rexaud $(0),$.23 , rue Sainte-Sophie, a Versailles.

COMMISSAIRES DES COMPTES

M. Bondas (G.), 99, rue de Mogador, Paris ( $8 *^{*}$.

M. Pinson (Victor), 28, rue Guillaume-Tell, Paris (17*).

DIRECTIOX

Administralion centrale.

M. BÉgeÉ, Directeur des Exploitations, 93, rue de Rennes, Paris (16*).

M. Lheureux (Jules), Secrélaire général, 3 , rue Muncey, Paris $\left(9^{*}\right)$

M. Deschénes (J.-B.), Chef de la Comptabilité génêrale, 29 , boulevard Murat, Paris (16\%).

M. Gèrand, Ingènieur chef des services techniques, 9, rue Trétaigne, Paris (18*).

DIRECTEURS DE3 RÉSEAUX

M. LAcroix, 115, rue Jules-Lecesne, Le Harre.

M. Avris, 6, rue Sónac, Marseille.

M. Callot, avenue de Boumers, à Nancy.

M. Delavengsas, 179, faubourg Bannier, Orléans.

ORGAXISATIOX FINANCiÈnE

Services financiers: Comptoir National d'Escompte, 14, rue Bergère, Paris (9e) Société Gémérale, 29 , boulevard Haussmann, Paris $\left(9^{\circ}\right)$

Crédit Industriel et Commercial, 66, rue de la Victoiro, Paris $(9 \cdot)$.

Société Marseillaise, 4 , rue Auber, Paris (9*)

Crédit Lyonnais, 19, boulevard des Italiens, Paris (9*)

Capital-actions: $\mathbf{1 1 0 , 0 0 0}$ actions do 500 francs, soit $55,000,000$ de francs. 


\section{GÉNÉRALE TRANCAISE DE TRAMWAYs}

Emprunt-obligations : $\mathbf{1 4 0 . 0 0 0}$ obligations de 500 francs $4 \%$, autorisées par décisions ministérielles des 4 avril 1906, 15 juin 1908,20 mai 1911,3 arril 1914, remboursables au pair, de 1919 a 1962 . - Coupon 15 juin et 15 décembre. 17.840 de ces titres étaient amortis au 1*r janvier 1926.9 .131 obligations di 500 francs $30 / 0$, dont 407 amorties au $t^{* r}$ janvier 1926.

Admission à la cote à la Bourse de Paris, au comptant.

Répartition des bénéfices: $1^{\circ}, 5 \%$ à la réserve lègale jusqu'a ce qu'elle ait atteint $1 / 10$ du capital social

2. Intérét de $5 \%$ aux actions, a titre de premier dividende.

Le solde réparti : $20 \%$ au Conseil d'administration, $80 \%$ aux actionnaires.

Repartitions effectues:

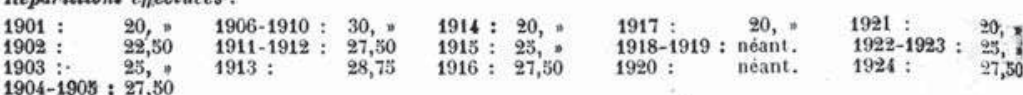

1904-1905 : 27,50

Cours moyen des actions :

$\begin{array}{ll}1910: 599,56 & 1913: 504,16 \\ 1911: 572,61 & 1914: 502,82\end{array}$

$\begin{array}{ll}1911: 572,61 & 1914: 302,82 \\ 1912: 524,02 & 1915: 390,69\end{array}$

$1917: 416,60$

$1922: 345$, ,

$1915: 390,69 \quad 1918: 381.83$

$1921: 249$,

$1923: 502, *$

Cours moyen des obligations $40 / 0$ :

$1910: 492,05 \quad 1913: 440,30$

$1911: 483,40 \quad 1914: 436,78$

$1916: 389,10$

$1917: 379,50$

$1919: 355,28$

$\begin{array}{lll}1917: 379,50 & 1920: 312, * & 1923: 319, * \\ 1918: 370,24 & 1921: 284, \text { : } & 1924: 266,89\end{array}$

$\begin{array}{lll}1917: 379,50 & 1920: 312, * & 1923: 319, * \\ 1918: 370,24 & 1921: 284, * & 1924: 266,89\end{array}$

1922 : 318 ,

Bilan au 81 Decembre 1925

Actif.

1*r établissement.............. 127.906.082,91

Travaux différés . . . . . . . . . . . . 37.692.944,03

- en cours.

$806.637,64$

1.135. 364,10

8.899.720,89

17.103 .954 .99

14. $249.974,39$

$8.102 .472,45$

$3,995,441,46$

$2.200 .000, *$

1.100.000,

$23.315 .598,51$

Fonds de reconstitution du Capit

Acompte sur le dividende de l'exereice.
Passif.

Capital-actions............... 55.000.000, Obligations................. 57.702 .420 , Emprunts des autorités concédantes. 52.890.393,83 Réserves................... 8.993.064,16 Amortissement obligations......... 8.651.460, Créditeurs et Compte d'ordre....... 15.508.384,58 Obligations à rembourser et coupons.. $\quad \mathbf{2 . 0 5 0 . 0 7 4 ,}$ Recinstitution du Capital......... $3.995 .441,40$ Dotation approvisionn. Rèseaux ... 14.249.974.39 Benefices reportes............... $258,680,51$

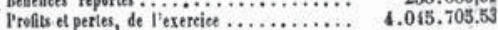

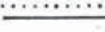

223.345 .598 .61

Profits et Pertes :

Débit.

Intérêts sur obligations.

Obligations amorties

Bmprunts autorités concéda........

Bènéfices de l'exercice.
2. $\{90,821$,

728.000,

$4.318,924,67$

$4.318 .924,67$
$4.045 .705,53$

$1.583 .451,20$
Crëdit.

Rémunérations et allocations diverses. 10.163.305, $\mathrm{B}$ Intérèts et produits divers ........ 1.490.146,20

$11,583,451,20$

Répartition béneficiaire :

Reconstitution du capital .......... 55.000 ,

Reserve lesale du capital $156.959,1$

Intérét $50 / 0$ aux actions............. $9.750 .000, n$

$\begin{array}{ll}\text { Conseil d'Administration ........... } & \begin{array}{l}216.750,67 \\ \text { Dividende aux actions ............ }\end{array} 275000,\end{array}$

Dividende aux actions ...............
Impót sur le revenu...............

Report à nouveau................. $186.658,89$

4. 30 i. 386,0 


\title{
Annexe 3
}

\section{Un groupe aux activités diversifiées}

\author{
Source : Annuaire des chemins de fer et des tramways, 1927
}

OMNIUNI LXONNA IS

\section{OMNIUM LYONNAIS}

Socielte anonyme, constituée le 17 janvier 1896 , pour 75 ans.

Silge social : 8 , rue de la Bourse, Lyon.

Dirction et administration centrale : 20, rue d'Athènes, Paris (9๑). Tél. : Central 08-72, 27-07 et 27-67. Tétégr.: Omnitram-Paris.

\author{
DOCEMENTATION OFTICIELLE
}

Statuts dóposés chez M* Bernard, notaire à Lyon, le 31 décembre 1895, modifiés les 4 juillet 1896, 31 aoùt et 10 novembre 1897,18 aoùt et 29 septembre 1898,7 novembre 1899,12 mars 1900 , 12 janvier 1903 , 27 novembre 1920 .

objet social: Toutes opérations industrielles, commerciales, immobilières et financières, et spécialement celles qui se rattactent à l'Industrie des Chemins de fer et Tramways.

$$
\begin{aligned}
& \text { La société exploite : } \\
& \text { Tramways d'A vignon }
\end{aligned}
$$

$\begin{array}{lll}\text { Lays d'A vignon } & 18 \mathrm{~km} .766 . \\ \text { - } & \text { de Bourges } & 8 \mathrm{~km} .800 . \\ \text { - de Cannes } & 21 \mathrm{~km} . \\ \text { - } & \text { de Cette } & 8 \mathrm{~km} .650 . \\ \text { - } & \text { de Fontainebleau } 5 \mathrm{~km} .400 . \\ \text { - } & \text { de Pau } & 6 \mathrm{~km} .900 . \\ \text { - } & \text { de Poitiers } & 6 \mathrm{~km} . \\ & \text { de Troyes } & 11 \mathrm{~km} .500 .\end{array}$

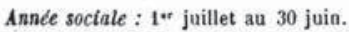

Assemblé generale: En novembre ou dècembre, composée des actionnaires propriétaires de 20 actions.

\section{CONSEIL. D'ADMISISTRATIOX}

3 a 11 membres nommés pour 6 ans, propriétaires de 50 actions :hacun.

Président : $\quad$ M. Perır (Joseph), 41, rue de Pétrograd, Paris (8\%). Tól. : Gutenberg 14-73.

Vice-Président, Administ,-déléguẻ : M. Magsıs (Pierre de), 9, boulevard Poreire, Paris (17*). Tél. : Wagram '56-86.

Administrateur : $\quad$ M. Bexedetrt (C* Fernand, 14, rue Royale, à Fontainebleau. Tèl. 1-77.

M. Fourtov (Adrien de), 25, avenue Charles-Floquet, Paris $(7 *$ ).

M. Frévil.te de Lorye (Charles de), 12, rue Cassette, Paris (6\%). Fleurus 52-74.

M. Lewandowski (Maurice) 7t, rue d'Assas, a Paris (6*). Tél. : Fleurus 05.63.

M. Chasaténes (Maurice), 152 , rue Paradis, Marseille.

M. Schweıscuth (Pierre), 3̆. rue de Varenne, Paris. Töl. : Fleurus 37-99.

M. Piatox (René), 49, rue de la Bourse, a Lyon.

M. Yvav (Elmond), 67, rue de Tocqueville, Paris (17*).

SERVICES TECHXIQUES ET ADMIXISTRATIFS.

M. Sekutowicz (Ladislas), Directeur des Services Techniques, 17, r. do Baudreuil, Meudon (S.-et-0.).

M. Brcavis (Henry), Directeur de la Comptabilité, 20, rue d"Athènes, Paris.

M. Roux (Stéphano). Secrétaire Genéral, 19, avenue Emile-Deschanel, Paris (7•). Tèl. : Sègur $38-15$.

M. Clémexr, Ingéniour en Chef des Exploitations.

M. GuÉar, - - des Services Blectriques.

M. Gıрот, - $\quad$ - de la Voie et des Batiments. 


\section{ORGANISATION FiNaXciéRE}

Capilal-aclions : 100.000 actions de $100 \mathrm{fr}$. soit 10.000 .000 de francs.

A lorigine, 7.500 actions de $100 \mathrm{fr} .=750.000 \mathrm{fr}$.

Porté en juillet et aoút $1896 \mathrm{à} \quad 1.500 .000 \mathrm{fr}$.

- en aoĉt et novembre 1897 à $\mathbf{4 . 0 0 0 . 0 0 0 ~ d e ~ f r . ~}$

- en aoùt et septembre 1898 a 10.000 .000 de fr.

- en mars 1900 a $\quad 20.000 .000$ de fr.

- en mars 1900 a 20.000 .000 de fr.

L'Assemblée du 12 janvier 1903 a réduit le capital à 10.000 .000 de fr. par l'échange de deux actions anciennes contre uso nouvelle.

II a été cré é outro 5.000 parts de fondateur.

Admission à la cote à la Bourse de Paris.

Repartition des bénéfices : $1^{*} 5 \%$ à la rèserve lègale jusqu'au quart du capital. 2. - Intérèt $6 \%$ aux actions non amorties.

3. - Prélèvements pour fonds d'amortissement, réserve facultative, ete.

Sur le-surplus : $15 \%$ au Gonseil d'admiuistration.

Le sulde réparti : $25 \%$ aux parts de fondateur, $75 \%$ aux actionnaires.

Repartitions effectuées :

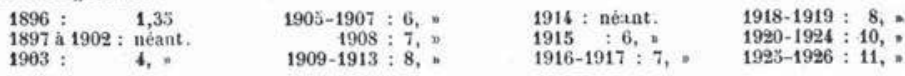

Cours moyen des actions :

$\begin{array}{llll}1911: 159,89 & 1915: 93,39 & 1919: 133,62 & 1923: 152,20 \\ 1912: 151,59 & 1916: 103,23 & 1920: 136, * & 1924: 142,63 \\ 1913: 163,36 & 1917: 118,17 & 1921: 137, \cdots & 1925: 125,65 \\ 1916: 135,33 & 1918: 129,87 & 1922: 126, \cdots & \end{array}$

ExErcicz 1923-19z6 (Assemblée génêrale du 27 Novembre 1925).

Actif.

Immeuble et terrains

Caisse et banques.

Debiteurs diver.

Port-feuille....

.............. \$. 342.802,

Frais de premier

bilier divers a coutissement, mo

$\frac{1,}{20.017 .890,81}$
Passif.

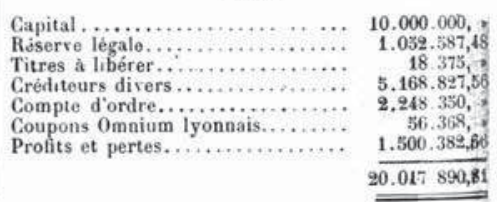

Crédit.

Dèbit.

Profits et Pertes :

Frais génèraux .. ............ 743.055 .02 1. $500.38 z, 66$

2.243.427,68 Produits de l'exercice. Report de lexercice précèdent....

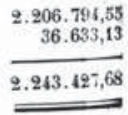

$206.794,55$ $.243 .427,68$

Répartition des béuifices.

Réserve légale.

Dividende $6 \%$

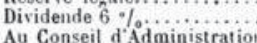

Dividende supplementaire 5 .

Aur parts de fondateurs.

Report i nouve tu.

$73.187,67$

600.000 ,

118..586, 36

300.000 ,

166.166,65

$166.166,03$
$41.944,23$ 
